\title{
Multidrug resistance in clinical isolates of Stenotrophomonas maltophilia: roles of integrons, efflux pumps, phosphoglucomutase (SpgM), and melanin and biofilm formation
}

\author{
S.-J. Liaw ${ }^{\mathrm{a}, \mathrm{b}}$, Y.-L. Lee ${ }^{\mathrm{a}}$, P.-R. Hsueh ${ }^{\mathrm{b}, \mathrm{c}, *}$ \\ a Department and Graduate Institute of Clinical Laboratory Sciences and Medical Biotechnology, National Taiwan University College of Medicine, Taipei, Taiwan \\ ${ }^{\mathrm{b}}$ Department of Laboratory Medicine, National Taiwan University Hospital, National Taiwan University College of Medicine, Taipei, Taiwan

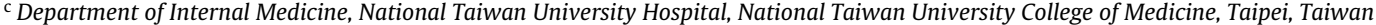

\section{A R T I C L E I N F O}

\section{Article history:}

Received 15 May 2009

Accepted 9 September 2009

\section{Keywords:}

Stenotrophomonas maltophilia

Multidrug resistance

Integrons

Efflux pumps

Phosphoglucomutase (SpgM)

Melanin

Biofilm formation

\begin{abstract}
A B S T R A C T
Integrons, efflux pumps, phosphoglucomutase (SpgM), and melanin and biofilm formation were investigated in 40 multidrug-resistant (MDR) and 30 non-MDR Stenotrophomonas maltophilia isolates recovered from patients treated at National Taiwan University Hospital (Taipei, Taiwan). Class 1 integrons were clearly associated with multidrug resistance. Sequencing data revealed that aminoglycoside-modifying

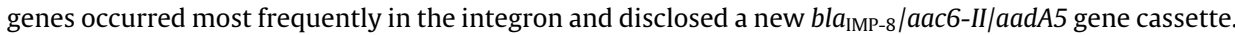
Semi-quantitative reverse transcriptase polymerase chain reaction (RT-PCR) was performed to assess the expression of Sme efflux pumps and SpgM in S. maltophilia. MDR isolates exhibited hyperexpression of SmeABC, and SmeDEF and SpgM were more frequently found in MDR isolates than non-MDR isolates. In addition, the ability of MDR isolates to form melanin-like pigment and biofilm was also greater than that of the non-MDR isolates. The SmeABC or SmeDEF pump was shown to be associated with resistance to all agents tested. The presence of an integron as well as production of pigment and biofilm was also responsible for resistance against eight, six and six of the tested agents, respectively. High SpgM expression was associated with resistance to only three of the tested agents. These findings define the important roles of integrons, efflux pumps, and melanin-like pigment and biofilm formation in the multidrug resistance of $S$. maltophilia. MDR isolates possessed more resistance mechanisms than susceptible strains.
\end{abstract}

(C) 2009 Elsevier B.V. and the International Society of Chemotherapy. All rights reserved.

\section{Introduction}

Stenotrophomonas maltophilia, a non-fermentative Gramnegative bacillus, is one of the multiresistant opportunistic nosocomial pathogens especially affecting immunocompromised patients and is being isolated worldwide with increasing frequency [1-4]. Clinical isolates of $S$. maltophilia are often highly resistant to most of the currently used antimicrobial agents, including carbapenems, and therapy of S. maltophilia infection presents a significant challenge both for clinicians and microbiologists [2-4]. A variety of antimicrobial resistance determinants have been reported in $S$. maltophilia, including tripartite efflux pumps (SmeDEF and SmeABC), integrons and phosphoglucomutase (SpgM) [1,5-7]. A role in multidrug resistance has been demonstrated for SmeDEF, and other efflux pumps are also known to be involved in resistance but only in some $S$. maltophilia isolates $[8,9]$. Integrons comprise an integrase-encoding gene that allows

\footnotetext{
* Corresponding author. Tel.: +88622312 3456x65355; fax: +886223224263. E-mail address: hsporen@ntu.edu.tw (P.-R. Hsueh).
}

for site-specific insertion of resistance gene cassettes between two highly conserved adjacent nucleotide sequences $\left[3^{\prime}\right.$ conserved segment $\left(3^{\prime} \mathrm{CS}\right)$ and $5^{\prime}$ conserved segment $\left.\left(5^{\prime} \mathrm{CS}\right)\right]$. Integrons are located on transposons or plasmids that facilitate the rapid spread of integrons to other strains and bacterial species [10-12]. SpgM, a homologue of AlgC in Pseudomonas aeruginosa associated with lipopolysaccharide (LPS) and alginate biosynthesis, was shown to play a role in virulence and antibiotic resistance of $S$. maltophilia [7]. Melanin-like pigment and biofilm formation were generally implicated as common causes of antimicrobial resistance [13-15].

In the present study, we assessed the roles of integrons, efflux pumps, SpgM, melanin and biofilm on multidrug resistance among clinical isolates of $S$. maltophilia.

\section{Materials and methods}

\subsection{Bacterial isolates}

Forty clinical multidrug-resistant (MDR) [i.e. resistant to five or more of the six categories of drugs tested, including cephalosporins and $\beta$-lactams plus $\beta$-lactamase inhibitors, carbapenems, 
monobactams, aminoglycosides, quinolones and trimethoprim/sulfamethoxazole (SXT)] and 30 non-MDR (i.e. resistant to four or less of the six categories of drugs tested) S. maltophilia isolates were collected at National Taiwan University Hospital (Taipei, Taiwan) during 2002-2003. These isolates were obtained from sputum $(n=23)$, wounds $(n=2)$, central venous catheter $(n=1)$, urine $(n=8)$, blood $(n=34)$, cerebrospinal fluid $(n=1)$ and eye $(n=1)$.

All of the isolates were identified by conventional biochemical identification methods and were confirmed by the API $20 \mathrm{E}$ (bioMérieux, La-Balme-les-Grottes, France) and Vitek automated systems (bioMérieux Vitek, Hazelwood, MO). Random amplified polymorphic DNA (RAPD) analysis (Operon Technologies, Inc., Alameda, CA) was used to exclude repeat isolates [1].

\subsection{Antimicrobial susceptibility testing}

Antimicrobial susceptibilities were determined by the agar dilution method as described by the Clinical and Laboratory Standards Institute [16,17]. The following agents were included: gentamicin (GEN); ciprofloxacin (CIP) and levofloxacin (LEV); SXT; ceftazidime (CAZ) and cefepime (FEP); ticarcillin/clavulanic acid (TIM) and piperacillin/tazobactam (TZP); aztreonam (ATM); and meropenem (MER). Control strains for susceptibility testing included Escherichia coli ATCC 25922, P. aeruginosa ATCC 27853 and Staphylococcus aureus ATCC 29213.

\subsection{Integron polymerase chain reaction (PCR)}

Class 1 integrons were detected with class I integrasespecific primers (5'-ACATGTG ATGGCGACGCACGA-3' and 5'ATTTCTGTCCCTGGCTGGCGA-3'). Gene cassettes within integrons were amplified with primers specific for the integron $5^{\prime} \mathrm{CS}\left(5^{\prime}\right.$ GGCATCCAAGCAGCAAG-3') and 3'CS (5'-AAGCAGACTTGACCTGA$3^{\prime}$ ) [18]. Template DNA for PCR was prepared as described by Ploy et al. [18]. The amplicons were then sequenced and sequence com- parisons were made using the BLAST program. Plasmid DNA was extracted by the method of Kado and Liu [19].

\subsection{Semi-quantitative reverse transcriptase polymerase chain reaction $(R T-P C R)$}

Cells were prepared and inoculated onto a Mueller-Hinton agar plate as in the agar dilution method $[16,17,20]$. Following overnight culture, cells were collected to make a $1.5 \mathrm{~mL}$ suspension of optical density at $550 \mathrm{~nm}\left(\mathrm{OD}_{550 \mathrm{~nm}}\right)=1.0$. RNA was prepared using an RNA-Bee ${ }^{\mathrm{TM}}$ Kit (Tel-Test Inc., Friendswood, TX) and cDNA was obtained with the SuperScript ${ }^{\mathrm{TM}}$ First-Strand Synthesis System for RT-PCR (Invitrogen Corp., CA) using random hexamers. Primer pairs 5'-GTCGACCTG GTACAGCA-3'/5'-ACCTTAACCTGTGCCTTG-3', 5'CCAAGAGCCTTTC CGTCAT-3' $/ 5^{\prime}$-TCACGCTGAAGTCCGAGA-3' and 5'-GTGACTTCGACC GTTGCTTC-3'/5'-ATCTTTTCCTTGATGAACGC-3' were used for PCR to detect the expression of smeA, smeD and spgM, respectively, using cDNA of $16 \mathrm{~S}$ rRNA as an internal control. Four micrograms of total RNA was reverse transcribed to cDNA. The RNA was then denatured by the addition of $\mathrm{NaOH}$ and incubation at $65^{\circ} \mathrm{C}$ for $10 \mathrm{~min}$ and $1 \mu \mathrm{L}$ of $\mathrm{cDNA}$ solution was used as the template for PCR. PCR was performed with a 10 min denaturation step at $95^{\circ} \mathrm{C}$ followed by 25 cycles (cDNA of $16 \mathrm{~S}$ rRNA), 30 cycles (smeD) or 35 cycles (smeA and spgM) of $1 \mathrm{~min}$ at $95^{\circ} \mathrm{C}$ and $1 \mathrm{~min}$ at $55^{\circ} \mathrm{C}$ (for $s m e D$ ) or $65^{\circ} \mathrm{C}$ (for cDNA of $16 \mathrm{~S}$ rRNA, smeA and $s p g M$ ) for annealing and extension. Gel images were digitally captured with a CCD camera and band intensity was analysed using Image software (http://rsb.info.nih.gov/ij/). The relative expression quantity was evaluated by the ratios of band intensity to cDNA of 16S rRNA for three independent experiments. Isolates with a ratio greater than the average ratio of all isolates were designated as high expression, and those with equal to or less than the average ratio were designated as low expression. A number of different cycle numbers and template concentrations were used to confirm linearity within the experiment.

Table 1

Antibiotic susceptibility profiles of multidrug-resistant (MDR) and non-MDR Stenotrophomonas maltophilia isolates.

\begin{tabular}{|c|c|c|c|c|c|c|}
\hline \multirow[t]{2}{*}{ Antimicrobial agent } & \multicolumn{3}{|l|}{$\operatorname{MDR}(n=40)$} & \multicolumn{3}{|c|}{ Non-MDR $(n=30)$} \\
\hline & $\mathrm{MIC}_{90}(\mu \mathrm{g} / \mathrm{mL})$ & $\% \mathrm{~S}(n)$ & $\% \mathrm{R}(n)$ & $\mathrm{MIC}_{90}(\mu \mathrm{g} / \mathrm{mL})$ & $\% \mathrm{~S}(n)$ & $\% \mathrm{R}(n)$ \\
\hline CAZ & $>256$ & $5(2)$ & $95(38)$ & $>256$ & $83(25)$ & $17(5)$ \\
\hline FEP & 256 & $2.5(1)$ & $98(39)$ & 64 & $73(22)$ & $27(8)$ \\
\hline TIM & $128 / 2$ & $63(25)$ & $38(15)$ & $16 / 2$ & $100(30)$ & $0(0)$ \\
\hline TZP & $>256 / 4$ & $18(7)$ & $83(33)$ & $>256$ & $80(24)$ & $20(6)$ \\
\hline MER & $>256$ & $2.5(1)$ & $98(39)$ & 256 & $20(6)$ & $80(24)$ \\
\hline ATM & $>256$ & $2.5(1)$ & $98(39)$ & 256 & $23(7)$ & $77(23)$ \\
\hline GEN & $>256$ & $10(4)$ & $90(36)$ & 64 & $30(9)$ & $70(21)$ \\
\hline CIP & 128 & $0(0)$ & $100(40)$ & 8 & $83(25)$ & $17(5)$ \\
\hline LEV & 64 & $33(13)$ & $68(27)$ & 8 & $90(27)$ & $10(3)$ \\
\hline SXT & $16 / 304$ & $58(23)$ & $43(17)$ & $2 / 38$ & $100(30)$ & $0(0)$ \\
\hline
\end{tabular}

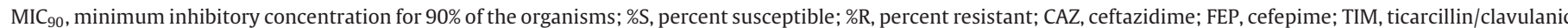
acid; TZP, piperacillin/tazobactam; MER, meropenem; ATM, aztreonam; GEN, gentamicin; CIP, ciprofloxacin; LEV, levofloxacin; SXT, trimethoprim/sulfamethoxazole.

Table 2

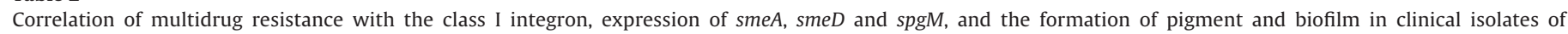
Stenotrophomonas maltophilia.

\begin{tabular}{|c|c|c|c|c|c|c|c|c|c|c|c|}
\hline & \multicolumn{2}{|c|}{ Class I integron } & \multicolumn{2}{|l|}{ SmeA } & \multicolumn{2}{|l|}{ SmeD } & \multicolumn{2}{|l|}{ SpgM } & \multicolumn{2}{|l|}{ Pigment } & \multirow{2}{*}{$\begin{array}{l}\text { Biofilm } \\
\text { Mean (S.D.) } \mathrm{OD}_{540 \mathrm{~nm}}\end{array}$} \\
\hline & + ve $(n=42)$ & -ve $(n=28)$ & $\mathrm{H}(n=29)$ & $\mathrm{L}(n=41)$ & $\mathrm{H}(n=44)$ & $\mathrm{L}(n=26)$ & $\mathrm{H}(n=33)$ & $\mathrm{L}(n=37)$ & $\mathrm{H}(n=38)$ & $\mathrm{L}(n=32)$ & \\
\hline $\operatorname{MDR}(n=40)$ & $33(83)^{\mathrm{a}}$ & $7(18)$ & $24(60)$ & $16(40)$ & $34(85)$ & $6(15)$ & $23(58)$ & $17(43)$ & $30(75)$ & $10(25)$ & $0.52(0.34)$ \\
\hline Non-MDR $(n=30)$ & $\begin{array}{l}9(30) \\
P<0.01^{\mathrm{b}}\end{array}$ & $21(710)$ & $\begin{array}{l}5(17) \\
P<0.01^{b}\end{array}$ & $25(83)$ & $\begin{array}{l}10(33) \\
P<0.01^{\mathrm{b}}\end{array}$ & $20(67)$ & $\begin{array}{l}10(33) \\
P<0.05^{b}\end{array}$ & $20(67)$ & $\begin{array}{l}8(27) \\
P<0.01^{b}\end{array}$ & $22(73)$ & $\begin{array}{l}0.15(0.13) \\
P<0.01^{\mathrm{c}}\end{array}$ \\
\hline
\end{tabular}

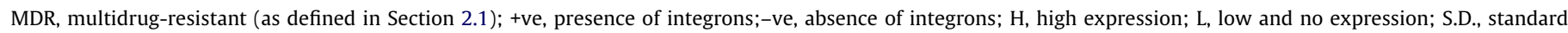
deviation; $\mathrm{OD}_{540 \mathrm{~nm}}$, optical density at $540 \mathrm{~nm}$.

a Percentage of MDR or non-MDR isolates in parentheses.

b Comparison between MDR and non-MDR isolates by Fisher's exact test.

c Comparison between MDR and non-MDR isolates by Student's $t$-test. 


\subsection{Biofilm assay}

The biofilm assay was performed as described previously [14]. Overnight Luria-Bertani cultures were transferred to the wells of polystyrene microtitre plates. The plates were incubated at $37^{\circ} \mathrm{C}$ for $10 \mathrm{~h}$ and then stained with crystal violet. Solubilised crystal violet was determined.

\subsection{Pigment assay}

A melanin-like pigment assay was performed as described previously [21]. Bacteria were cultured in L-tyrosine-containing agar to facilitate the observation of brown pigment production. Pigment formation was recorded as $0,1+, 2+$ or $3+$, with increased intensity and intensity equal to or greater than $2+$ designated as high pigment expression.

\section{Results}

\subsection{Bacterial isolates and antimicrobial susceptibility}

The RAPD patterns of the 70 isolates were not identical, indicating the diversity of the isolates (i.e. not clonally related). The results of susceptibility testing (Table 1) showed that most of the S. maltophilia isolates were resistant to MER (90\%), ATM (89\%), GEN (81\%), FEP (67\%), CIP (64\%) and CAZ (61\%). Among the agents tested, MDR and non-MDR isolates exhibited minor differences in resistance to MER, ATM and GEN (98\% vs. $80 \%, 98 \%$ vs. $77 \%$ and $90 \%$ vs. $70 \%$, respectively). TIM and SXT (79\% and 76\% susceptible, respectively) were the most active drugs against $S$. maltophilia.

\subsection{Integrons}

Forty-two isolates (60\%) carried detectable class 1 integrons (integron I). More MDR isolates (83\%) possessed class 1 integrons than non-MDR isolates (30\%) (Table 2). Integron-positive isolates were significantly more likely to be resistant to CIP, CAZ, FEP, TIM, TZP, SXT, MER and GEN (Table 3). Gene cassettes within integrons included aacA4, aadB, aacC4, aacA6'-Ib, smr, smr/aacA4, qac, cmlA, catB2 and bla IMP-8/aac6-II/aadA5. Two isolates harboured plasmid DNA, but Southern blot assay demonstrated that neither of these two isolates carried a plasmid-mediated integron.

\subsection{Efflux pumps}

The rate of high smeD expression (63\%) was greater than the rate of high smeA expression (41\%) in the S. maltophilia isolates studied. MDR isolates expressed high SmeD and high SmeA more frequently than non-MDR isolates ( $85 \%$ vs. $33 \%$ for SmeD and $60 \%$ vs. $17 \%$ for SmeA) (Table 2). High smeA or smeD expression was correlated with resistance to all of the drugs tested (Table 3 ).

\section{4. $\operatorname{Spg} M$}

The difference in spgM expression between MDR and non-MDR isolates was less significant compared with the other determinants tested $(P<0.05)$ (Table 2$)$. High SpgM expression was correlated with three lactams (CAZ, TIM and TZP) only (Table 3 ).

\subsection{Melanin-like pigment}

Most MDR isolates produced a higher level of pigment than non-MDR isolates (75\% vs. 27\%) (Table 2). High-pigment-producing isolates exhibited significant correlation with resistance to CIP, LEV, CAZ, FEP, TIM and TZP (Table 3).

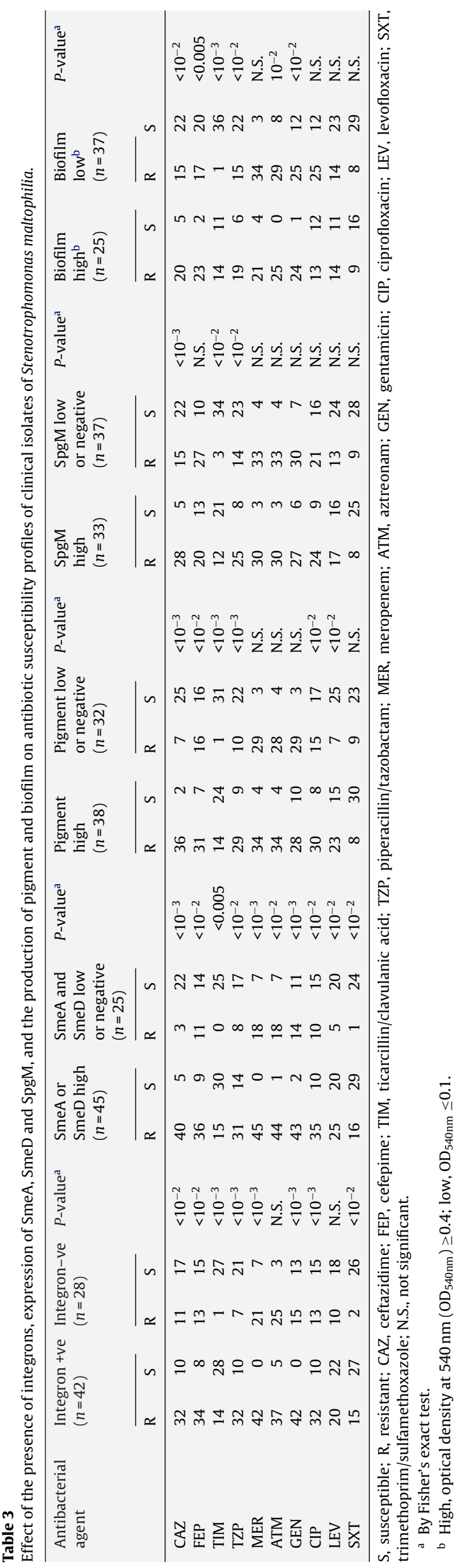




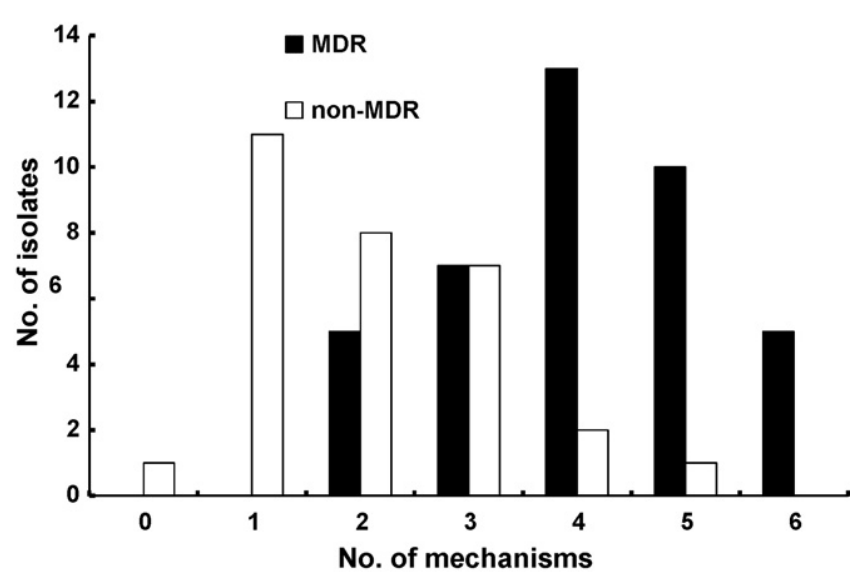

Fig. 1. Comparison of numbers of resistance mechanisms between multidrugresistant (MDR) and non-MDR Stenotrophomonas maltophilia isolates.

\subsection{Biofilm formation}

MDR isolates had an average $\mathrm{OD}_{540 \mathrm{~nm}}$ value of 0.52 compared with a value of 0.15 for non-MDR isolates $(P<0.01)$ (Table 2$)$. Biofilm formation was not correlated with resistance to CIP, LEV (both quinolones), SXT or MER (Table 3).

The data obtained in this study imply that all resistance determinants contribute to the MDR phenotype of S. maltophilia. Among them, the presence of efflux pumps and integron I may play important roles leading to multidrug resistance of $S$. maltophilia. Fig. 1 shows that 28 MDR isolates possessed over four mechanisms of resistance, whereas most non-MDR isolates possessed less than three mechanisms.

\section{Discussion}

Both combined specific resistance genes and multidrug resistance determinants have been described as conferring multidrug resistance in other Gram-negative bacteria [22-25]. It is expected that such mechanisms are likely to be present in S. maltophilia. In this study, we assessed the contribution of integrons, efflux pumps, SpgM, and melanin and biofilm formation to multidrug resistance of S. maltophilia. A partial correlation was found between the mechanisms studied and multidrug resistance-some were strong (i.e. integron I) and some were only weak (i.e. SpgM). Further experiments such as genetic knockouts should be performed to determine whether they are playing a role in multidrug resistance.

It has been shown that class 1 integrons in Gram-negative isolates from different hospitals are associated with decreased susceptibility to multiple antibiotic agents $[10,11,26]$. In this study, we found that integron I contributed to resistance against eight of the drugs tested. The spread of metallo- $\beta$-lactamases (MBLs) in Enterobacteriaceae is becoming a clinical concern in Taiwan and consistent and constant surveillance of MBL-producers should be the prime measure to prevent their further dissemination $[27,28]$. A kind of MBL, IMP-8, was previously identified from clinical isolates of Klebsiella pneumoniae and Enterobacter cloacae, and these

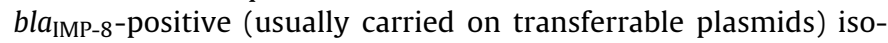
lates were all MDR $[27,28]$. The present study is the first to identify

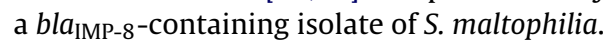

The origin of $b l a_{\text {IMP-8 }}$ remains to be determined by cloning its flanking sequences. None of the integron-positive isolates carried plasmids. The ability of $S$. maltophilia to be involved in genetic transfer and the acquisition of resistance determinants from other bacteria remains to be clarified. The integron I carriage rate in this study is higher than that reported by Chang et al. (60\% vs. 22\%) [1], which may due to the different origins of the samples collected. The finding that the most common gene cassette carried by integron I was comprised of aminoglycoside resistance determinants may account for the significant difference in susceptibility to GEN between the integron-positive and -negative isolates. The presence of an integron I was found to make a significant contribution to SXT resistance. Among the 17 SXT-resistant isolates, 15 (88\%) carried an integron I and 11 of them possessed sul1 genes. This finding confirms the increased incidence of integron I in SXT-resistant clinical isolates of S. maltophilia [29,30]. Both the use of quaternary ammonium compounds and triclosan in the natural environment have the potential to select for resistance to other antibiotics $[31,32]$. The frequent occurrence of the qac gene cassette within integron I in the present study implies that multidrug resistance may result from the introduction of biocides into hospital settings to prevent bacterial infections.

Because of its association with LPS synthesis, SpgM expression may affect the structure of the bacterial envelope and alter susceptibility to antimicrobial agents belonging to the $\beta$-lactam class that act on penicillin-binding proteins located in the periplasm. In this study, we found that high expression of SpgM in S. maltophilia was associated with resistance to CAZ, TZP and TIM but not to MER, ATM and FEP. We also found that most isolates that exhibited no significant correlation between high SpgM expression and resistance to MER, ATM and FEP expressed L1 or L2 $\beta$-lactamases. This implies that resistance to MER, ATM and FEP in SpgM high-expressed $S$. maltophilia might be due to expression of L1 or L2 $\beta$-lactamases.

The finding that SmeD expression was more important than SmeA expression in the MDR isolates studied here is contrary to the report of Chang et al. [1]. Different sample populations and the use of different gene targets (smeD vs. smeF and smeA vs. smeB) may account for this discrepancy [1].

Melanin-like pigment has been shown to protect cells from environmental insult in bacteria and fungi $[15,33]$. In this study, we found that pigment production in S. maltophilia was associated with antibiotic resistance. Our preliminary data on drug susceptibility testing using cells with and without pigment formation indicated that pigment-bearing cells were more resistant to antibiotics.

It is clear that biofilm-grown cells express properties distinct from those of planktonic cells, one of which is increased resistance to antimicrobial agents. The nature of the resistance generated by biofilms, despite extensive research, remains an enigma. We found that resistance to CIP and LEV was not associated with high biofilm-forming ability. This finding is consistent with a study by Di Bonaventura et al. [14], which disclosed that quinolones reduce adherence of $S$. maltophilia to plastic surfaces and can reduce preformed biofilms of $S$. maltophilia.

In conclusion, our findings define the important roles of integrons, efflux pumps, melanin-like pigment and biofilm formation in the multidrug resistance of $S$. maltophilia. MDR isolates possessed more resistance mechanisms than susceptible strains. Integron PCR or semi-quantitative RT-PCR for either SmeABC or SmeDEF might be a useful method of screening for MDR S. maltophilia.

Funding: This work was supported by grants (NSC-94-2320-B002-080) from the National Science Council and National Taiwan University Hospital, Taipei, Taiwan.

Competing interests: None declared.

Ethical approval: Not required.

\section{References}

[1] Chang LL, Chen HF, Chang CY, Lee TM, Wu WJ. Contribution of integrons, and SmeABC and SmeDEF efflux pumps to multidrug resistance in clinical isolates of Stenotrophomonas maltophilia. J Antimicrob Chemother 2004;53:518-21.

[2] Liaw SJ, Teng LJ, Hsueh PR, Ho SW, Luh KT. In vitro activities of antimicrobial combinations against clinical isolates of Stenotrophomonas maltophilia. J Formos Med Assoc 2002;101:495-501. 
[3] Tan CK, Liaw SJ, Yu CJ, Teng LJ, Hsueh PR. Extensively drug-resistant Stenotrophomonas maltophilia in a tertiary care hospital in Taiwan: microbiologic characteristics, clinical features, and outcomes. Diagn Microbiol Infect Dis 2008;60:205-10.

[4] Denton M, Kerr KG. Microbiological and clinical aspects of infection associated with Stenotrophomonas maltophilia. Clin Microbiol Rev 1998;11:57-80.

[5] Li XZ, Zhang L, Poole K. SmeC, an outer membrane multidrug efflux protein of Stenotrophomonas maltophilia. Antimicrob Agents Chemother 2002;46: 333-43.

[6] Zhang L, Li XZ, Poole K. SmeDEF multidrug efflux pump contributes to intrinsic multidrug resistance in Stenotrophomonas maltophilia. Antimicrob Agents Chemother 2001;45:3497-503.

[7] McKay GA, Woods DE, MacDonald KL, Poole K. Role of phosphoglucomutase of Stenotrophomonas maltophilia in lipopolysaccharide biosynthesis, virulence, and antibiotic resistance. Infect Immun 2003;71:3068-75.

[8] Gould VC, Avison MB. SmeDEF-mediated antimicrobial drug resistance in Stenotrophomonas maltophilia clinical isolates having defined phylogenetic relationships. J Antimicrob Chemother 2006;57:1070-6.

[9] Crossman LC, Gould VC, Dow JM, Vernikos GS, Okazaki A, Sebaihia M, et al. The complete genome, comparative and functional analysis of Stenotrophomonas maltophilia reveals an organism heavily shielded by drug resistance determinants. Genome Biol 2008;9:R74.

[10] Chang YC, Shih DY, Wang JY, Yang SS. Molecular characterization of class 1 integrons and antimicrobial resistance in Aeromonas strains from foodborne outbreak-suspect samples and environmental sources in Taiwan. Diagn Microbiol Infect Dis 2007;59:191-7.

[11] Gu B, Tong M, Zhao W, Liu G, Ning M, Pan S, et al. Prevalence and characterization of class I integrons among Pseudomonas aeruginosa and Acinetobacter baumannii isolates from patients in Nanjing, China. J Clin Microbiol 2007;45:241-3.

[12] Jones ME, Peters E, Weersink AM, Fluit A, Verhoef J. Widespread occurrence of integrons causing multiple antibiotic resistance in bacteria. Lancet 1997;349:1742-3.

[13] Claus H. Laccases and their occurrence in prokaryotes. Arch Microbiol 2003; 179:145-50.

[14] Di Bonaventura G, Spedicato I, D’Antonio D, Robuffo I, Piccolomini R. Biofilm formation by Stenotrophomonas maltophilia: modulation by quinolones, trimethoprim-sulfamethoxazole, and ceftazidime. Antimicrob Agents Chemother 2004;48:151-60.

[15] Gomez BL, Nosanchuk JD. Melanin and fungi. Curr Opin Infect Dis 2003;16: 91-6.

[16] Clinical and Laboratory Standards Institute. Methods for dilution antimicrobial susceptibility tests for bacteria that grow aerobically; approved standard. 6th ed. Document M7-A6. Wayne, PA: CLSI; 2005.

[17] Clinical and Laboratory Standards Institute. Performance standards for antimicrobial susceptibility testing. Eighteenth informational supplement. Supplemental Tables, M100-S18. Wayne, PA: CLSI; 2008.
[18] Ploy MC, Denis F, Courvalin P, Lambert T. Molecular characterization of integrons in Acinetobacter baumannii: description of a hybrid class 2 integron. Antimicrob Agents Chemother 2000;44:2684-8.

[19] Kado CI, Liu ST. Rapid procedure for detection and isolation of large and small plasmids. J Bacteriol 1981;145:1365-73.

[20] Meadus WJ. A semi-quantitative RT-PCR method to measure the in vivo effect of dietary conjugated linoleic acid on porcine muscle PPAR gene expression. Biol Proced Online 2003;5:20-8.

[21] Wang G, Aazaz A, Peng Z, Shen P. Cloning and overexpression of a tyrosinase gene mel from Pseudomonas maltophila. FEMS Microbiol Lett 2000;185:23-7.

[22] Gould VC, Okazaki A, Howe RA, Avison MB. Analysis of sequence variation among smeDEF multi drug efflux pump genes and flanking DNA from defined 16S rRNA subgroups of clinical Stenotrophomonas maltophilia isolates. J Antimicrob Chemother 2004;54:348-53.

[23] Nikaido H. Multidrug efflux pumps of Gram-negative bacteria. J Bacteriol 1996;178:5853-9.

[24] Nikaido H. Prevention of drug access to bacterial targets: permeability barriers and active efflux. Science 1994;264:382-8.

[25] Poole K. Efflux-mediated multiresistance in Gram-negative bacteria. Clin Microbiol Infect 2004;10:12-26.

[26] Martinez-Freijo P, Fluit AC, Schmitz FJ, Grek VS, Verhoef J, Jones ME. Class I integrons in Gram-negative isolates from different European hospitals and association with decreased susceptibility to multiple antibiotic compounds. J Antimicrob Chemother 1998;42:689-96.

[27] Yan JJ, Ko WC, Chuang CL, Wu JJ. Metallo- $\beta$-lactamase-producing Enterobacteriaceae isolates in a university hospital in Taiwan: prevalence of IMP-8 in Enterobacter cloacae and first identification of VIM-2 in Citrobacter freundii. J Antimicrob Chemother 2002;50:503-11.

[28] Yan JJ, Ko WC, Tsai SH, Wu HM, Wu JJ. Outbreak of infection with multidrugresistant Klebsiella pneumoniae carrying bla IMP-8 $_{\text {in }}$ in a university medical center in Taiwan. J Clin Microbiol 2001;39:4433-9.

[29] Barbolla R, Catalano M, Orman BE, Famiglietti A, Vay C, Smayevsky J, et al. Class 1 integrons increase trimethoprim-sulfamethoxazole MICs against epidemiologically unrelated Stenotrophomonas maltophilia isolates. Antimicrob Agents Chemother 2004;48:666-9.

[30] Toleman MA, Bennett PM, Bennett DM, Jones RN, Walsh TR. Global emergence of trimethoprim/sulfamethoxazole resistance in Stenotrophomonas maltophilia mediated by acquisition of sul genes. Emerg Infect Dis 2007;13. 559-65.

[31] Gaze WH, Abdouslam N, Hawkey PM, Wellington EMH. Incidence of class 1 integrons in a quaternary ammonium compound-polluted environment Antimicrob Agents Chemother 2005;49:1802-7.

[32] Sanchez P, Moreno E, Martinez JL. The biocide triclosan selects Stenotrophomonas maltophilia mutants that overproduce the SmeDEF multidrug efflux pump. Antimicrob Agents Chemother 2005;49:781-2.

[33] Coyne VE, al-Harthi L. Induction of melanin biosynthesis in Vibrio cholerae. Appl Environ Microbiol 1992;58:2861-5. 